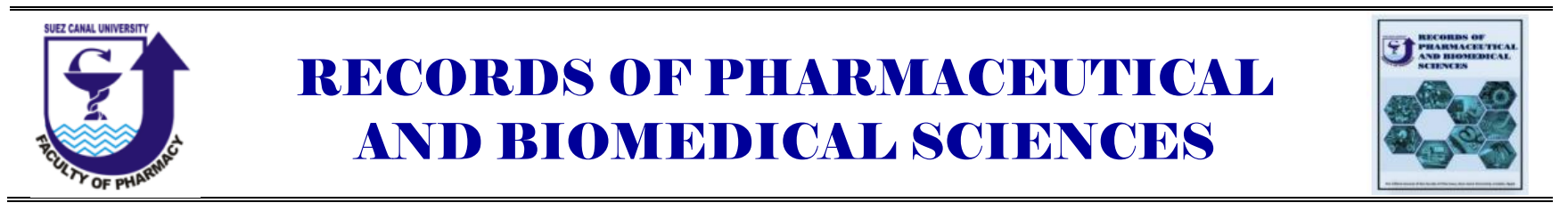

\title{
Role of Notch Signaling Pathway in Breast Cancer and Chemo-resistance
}

\author{
Esraa M. Mosalam ${ }^{a, *}$,Abdel-Aziz A. Zidan ${ }^{b, c}$, Eman T. Mehanna ${ }^{d}$, Noha M. Mesbah ${ }^{d}$, \\ Dina M. Abo-Elmatty ${ }^{d}$
}

\author{
${ }^{a}$ Biochemistry Department, Faculty of Pharmacy, Menoufia University, Egypt, ${ }^{b}$ Zoology Department, Faculty \\ of Science, Damanhour University, Egypt ${ }^{c}$ Center of Excellence in cancer Research (CECR), Tanta \\ University, Egypt, ${ }^{d}$ Biochemistry Department, Faculty of Pharmacy, Suez Canal University, Egypt
}

Received on: 11. 01. 2020

Revised on: 05. 02. 2020

Accepted on: 18. 02. 2020

Correspondence Author:

Tel: +201067802288

E-mail address:

esraa.mosalam@phrm.menofia.edu.eg.

\begin{abstract}
Despite the advanced treatment strategies, breast cancer remains the second cancer causing mortality across women worldwide. Notch signaling pathway has been found to be emerged in several cancers as well as in acquired and innate drug resistance. Therefore, understanding the role of Notch molecular mechanism could harbor beneficial impact to overcome chemoresistance and developing novel treatment strategies for cancer. Cisplatin (CIS) is an effective chemotherapeutic agent that is widely used against cancer but resistance is frequently occurs with limited therapeutic efficacy. One approach to overcome such unfavorable resistance is using complementary therapies with CIS. Thymoquinone (TQ) is a main compound in the essential oil of Nigella sativa. It has potent oncostatic activity by modulation of multiple regulatory pathways. Pentoxifylline (PTX) is a methylated xanthine derivative with remarkable anti-inflammatory and immunomodulatory actions. Combining TQ and PTX with CIS could be a promising strategy to suppress Notch signaling and overcome CIS resistance.
\end{abstract}

Keywords: Notch; Cisplatin; thymoquinone; Pentoxifylline;

Chemo-resistance

\section{Introduction}

Breast cancer is the second cancer causing mortality across women. GLOBOCAN 2018 estimated the new breast cancer cases to be $2,088,849$ with 626,679 deaths among women (Ferlay et al. 2019). Breast cancer incidence rate in Egypt were estimated based upon results from International Agency for Research on Cancer (IARC) and found to be about $47 \%$ with $18.8 \%$ mortality rate among women in 2018 (IARC 2018). Notch signaling is a highly preserved pathway that has a vital role in cellular differentiation, proliferation, renewal, and apoptosis (Ho et al. 2020). Latest studies proved implication of Notch in several types of cancers as well as in acquired and intrinsic drugresistance (Xiao et al. 2019). Cisplatin is a chemotherapeutic agent that was discovered in 1965 by Barnett Rosenberg and it is effectively used in treating several cancers. CIS belongs to platinum compounds, which act by binding to guanine bases on both strands of DNA (Muggia $\boldsymbol{e t}$ al. 2015). 
Thymoquinone (TQ) is the major active compound of the essential oil of Nigella sativa. Several studies outlined its effectiveness in hindering and treating cancer (Zidan et al. 2018). Besides, it has antiinflammatory, immunomodulatory, and anti-oxidant properties (Majdalawieh et al. 2015).

pentoxifylline (PTX) is a methylated xanthine derivative drug that was approved for peripheral vascular disorders (El-Haggar et al. 2018). PTX fights cancer through its apoptotic, anti-angiogenic, anti-metastatic, and immunomodulatory properties (Niderla-Bielińska et al. 2018)

\section{Molecular Notch signaling pathway}

\subsection{Canonical notch ligands}

In mammals, the canonical Notch pathway is encoded as four trans-membrane receptors (Notch 1-4) with five different trans-membrane ligands; delta-like ligand (DLL 1, 3, and 4) and Jagged ligand (JAG1 and JAG2) that are expressed by the adjacent cells (Ho et al. 2020) as presented in Figure (1).

When Notch extracellular domain (NECD) interacts with the ligand, disintegrin and metalloproteinase (ADAM) dislodges NECD (S2 cleavage) that continues the interaction with the ligand. Afterward, $\gamma$-secretase cleaves the remaining part of Notch receptor inside the cell (S3 cleavage) to release Notch intracellular domain (NICD) (Clara et al. 2019). Once NICD is free in the cytosol, it binds with various proteins including CBF1 suppressor of hairless and lag-1 (CSL), mastermind-like proteins (MamL), and P300. Subsequently, this entire complex translocates to the nucleus where P300 acts as histone acetylase and initiates expression of Notch-target genes as myelocytomatosis oncogene cellular homolog (Myc), hairy and enhancer of split-1 (Hes1), Hes related family BHLH transcription factor with YRPW Motif 1 (Hey1), cyclin D3, and P21 (Kovall et al. 2017)

\subsection{Non- canonical notch pathway}

The exact mediators of the non-canonical pathway are obscure. Consequently, understandng noncanonical Notch could afford valuable impact in several diseases' therapeutics. However and as shown in Figure (1), Wnt/ $\beta$-catenin pathway is a substantial regulator for Notch. The level of uncleaved membrane-bound Notch is inversely corrolated with active $\beta$-catenin (Andersen et al. 2012).

\subsection{Notch pathway and cancer}

Multiple documents proved the oncogenic role of Notch signaling in various human cancers, including but not limited to, lung, breast, ovarian, renal, endometria, and some hematological malignancies (Chatterjee et al. 2019). Notch pathway promotes tumorigenesis through conserving cancer stem cells (CSCs). These population of CSCs displayed higher activity of Notch signaling in comparison with their corresponding differentiated cells. Interestingly, the Notch $^{+}$cells showed ability to seed tumors unlike Notch $^{-}$cells, which failed to initiate tumors in xenograft mouse experiments (Butti et al. 2019).

Regulation of ligands' expression as well as the downstream effector genes not only plays a role in normal cellular responses but also in cancer development and progression (D'Souza et al. 2010). Each Notch ligand and targeted gene has a crucial function in promoting cancer as shown in Table (1) (Yuan et al. 2015).

\subsection{Notch-targeted therapies}

Designing of anti-Notch therapies is rapidly improving. The majority of Notch-targeted therapies aim to block the release of NICD from the membrane (Zlobin et al. 2019). As presented in Figure (2), there are several targets to block Notch signaling involving: ligands' expression, ligands' ubiquitination and trans-endocytosis, expression of Notch receptors' themselves, ligand-receptor binding, heterodimer detachment during Notch activation, ADAM cleavage of Notch, ubiquitination and endocytosis of $\gamma$-secretase substrate, $\gamma$-secretase cleavage of Notch, association of the coactivator complex with Notch and CSL, heterodimerization of Notch transcriptional complexes, Notch post-translational modifications, and expression of Notch-targeted genes (Clara et al. 2019).

\subsection{CIS and Notch Signaling}

Cisplatin is an alkylating chemotherapeutic agent that acts by different molecular mechanisms as shown in Figure (3). Basically, platinum atom of CIS is covalently binds to $\mathrm{N}^{7}$ position of purine residues in DNA strands causing intra- and interstrand crosslinks (Costa et al. 2019). The ability of 
Table (1): Role of Notch ligand/ target gene in carcinogenesis

Ligand/ target Function
gene

JAG1 Enhances angiogenesis

JAG2 Promote cell survival and proliferation

DLL1 Governing cell fate decisions and cell-to-cell communication

DLL3 Suppressing cell growth by induction of apoptosis

DLL4 Activating NF- $\mathrm{kB}$ signaling, which stimulates VEGF

Hes1 Sequence-specific DNA binding transcriptional factor involved in cellular proliferation and differentiation

Hey1 Development of neoplastic vasculature

NF-кB: nuclear factor kappa B and VEGF: vascular endothelial growth factor

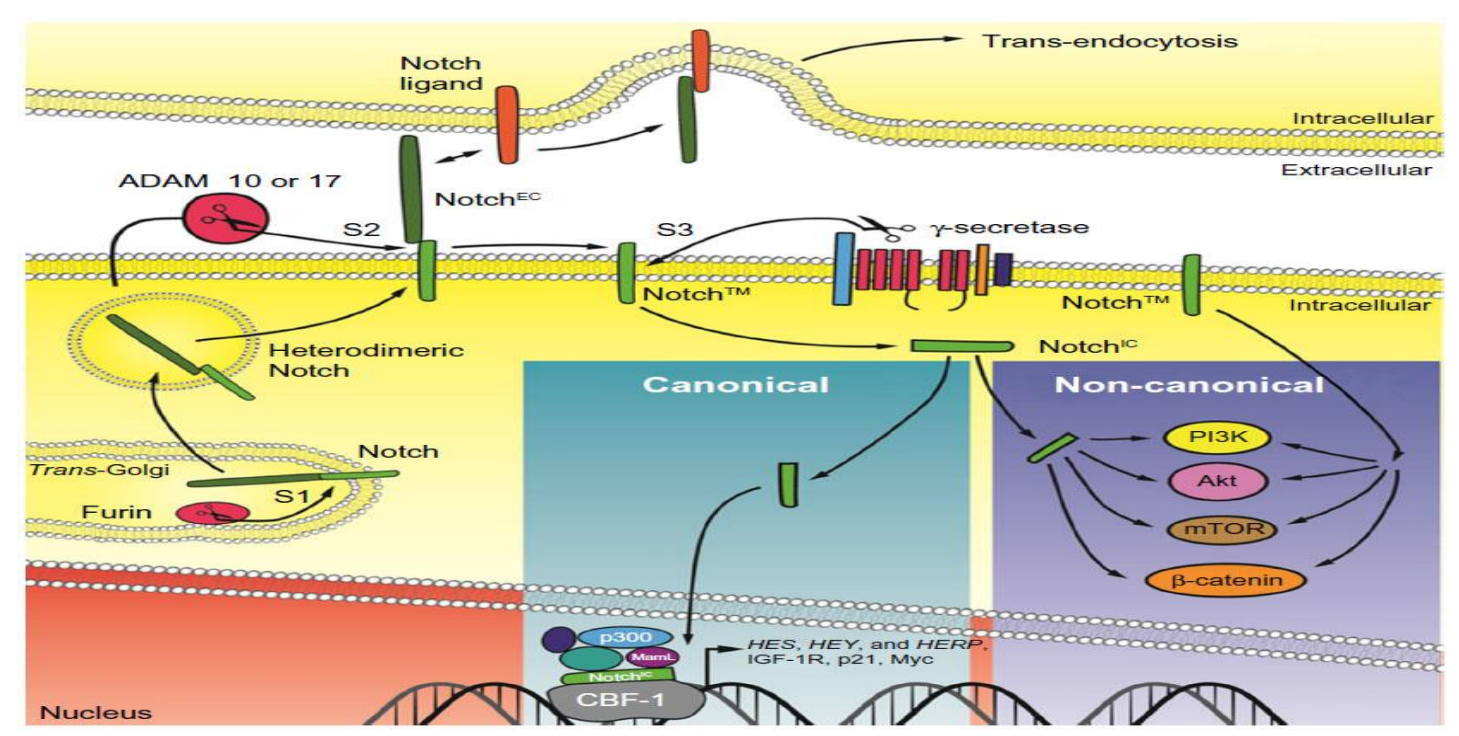

Figure (1): Canonical and non-canonical Notch signaling pathway. In Golgi apparatus, Notch receptor undergoes proteolytic cleavage mediated by furin proteases (S1 cleavage). The receptor is transported to the cell surface membrane. The extracellular domain of Notch (NECD) in the signaling cell binds with the ligands expressed by the neighboring cell. This induces S2 cleavage by ADAM, and releases NECD into the ligand-expressing cell. This is followed by the release of the Notch intracellular domain (NICD) that cleaved by $\gamma$ - secretase, which cleaves the NICD from the membrane by S3 and S4 cleavages. NICD traverses the nucleus and interacts with the CSL; results in the formation of an active complex with MamL and other co-activators and leads to the transcription of Notch target genes as HES and HEY. Non-canonical ligands activate Notch independently from CSL ligands .ADAM: disintegrin and metalloproteinase, MamL: Mastermind-like protein, CBF1: centromere-binding protein 1, CSL: CBF1 suppressor of hairless and lag-1, HES: hairy and enhancer of split-1, HEY: Hes related family BHLH transcription factor with YRPW motif 1, HERP: homocysteine-induced endoplasmic reticulum protein, IGF-1R: insulin-like growth factor 1 receptor, Myc: myelocytomatosis oncogene cellular homolog, Akt: protein kinase B, PI3K: phosphoinositide 3-kinase, mTOR: mechanistic target of rapamycin (Barse et al. 2015). 


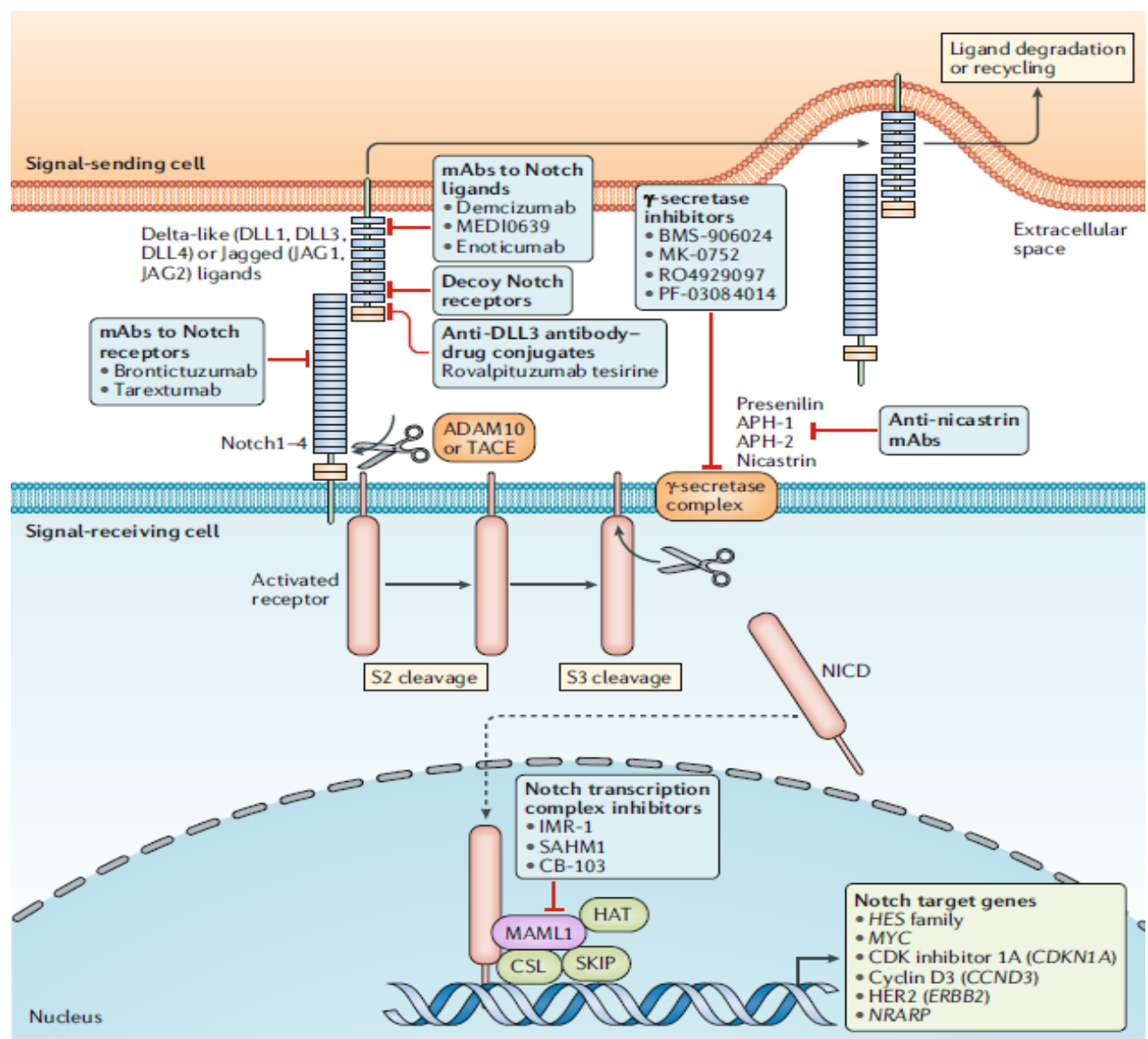

Figure (2): The canonical Notch pathway and related pharmacological inhibitors under investigation. Potential therapeutic agents targeting Notch pathway include monoclonal antibodies (mAbs) targeting Notch receptors or ligands, soluble decoys, ADAM inhibitors, $\gamma$ - secretase inhibitors, and inhibitors of NICD- interacting transcriptional regulators. NICD: Notch intracellular domain, ADAM10: disintegrin and metalloproteinase domaincontaining protein 10, TACE: tumour necrosis factor- $\alpha$ converting enzyme also known as ADAM17, APH: anterior pharynx- defective, HAT: histone acetyltransferase, MAML1: Mastermind- like 1, CSL: CBF1 suppressor of hairless and lag-1, SKIP: ski- interacting protein, HES: hairy and enhancer of split, MYC: myelocytomatosis oncogene cellular homolog, CDK: cyclin dependent kinase, ERBB2: receptor tyrosine-protein kinase, NRARP: Notch-regulated ankyrin repeat protein (Clara et al. 2019).

CIS to produce reactive oxygen species (ROS) is another cancer cell killing mechanism. The generated ROS stimulates extracellular signalregulated kinase (ERK), ataxia telangiectasia mutated (ATM), and P53 pathways. As a result, apoptosis is initiated and cancerous cell death is triggered (Cao et al. 2020). Moreover, CIS initiates apoptosis by inhibition of the anti-apoptotic B-cell lymphoma 2 (Bcl-2) and B-cell lymphoma-extra large (Bcl-xL), whereas it stimulates the proapoptotic Bcl-2-associated $\mathrm{X}$ protein (Bax) and caspase-9, which facilitates the release of cytochrome c (Qin et al. 2020).

Several in vitro and in vivo studies documented the bility of CIS to down-regulate Notch, JAG1, Hes1 (Tian et al. 2017), CTNNB1 with subsequent tumor-eradicating effect (Yin et al. 2018).
Unfortunately, by prolonged use of CIS, resistance of tumor cells toward CIS is frequently occurs. There are different mechanisms responsible for CIS resistance; one of them is reverse over-expression of Notch1 (Tian et al. 2017). There are several mechanisms to overcome resistance; giving CIS in a drug delivery system formulation, inhibition of metallothionein, using compination therapy with CIS (Achkar et al. 2018).

\subsection{Thymoquinone as a complementary therapy and Notch modulator}

Thymoquinone (TQ) is the fundamental active compound in the essential oil of Nigella sativa. Several studies documented its effectiveness in cancer therapy (Zidan et al. 2018). The antitumor 


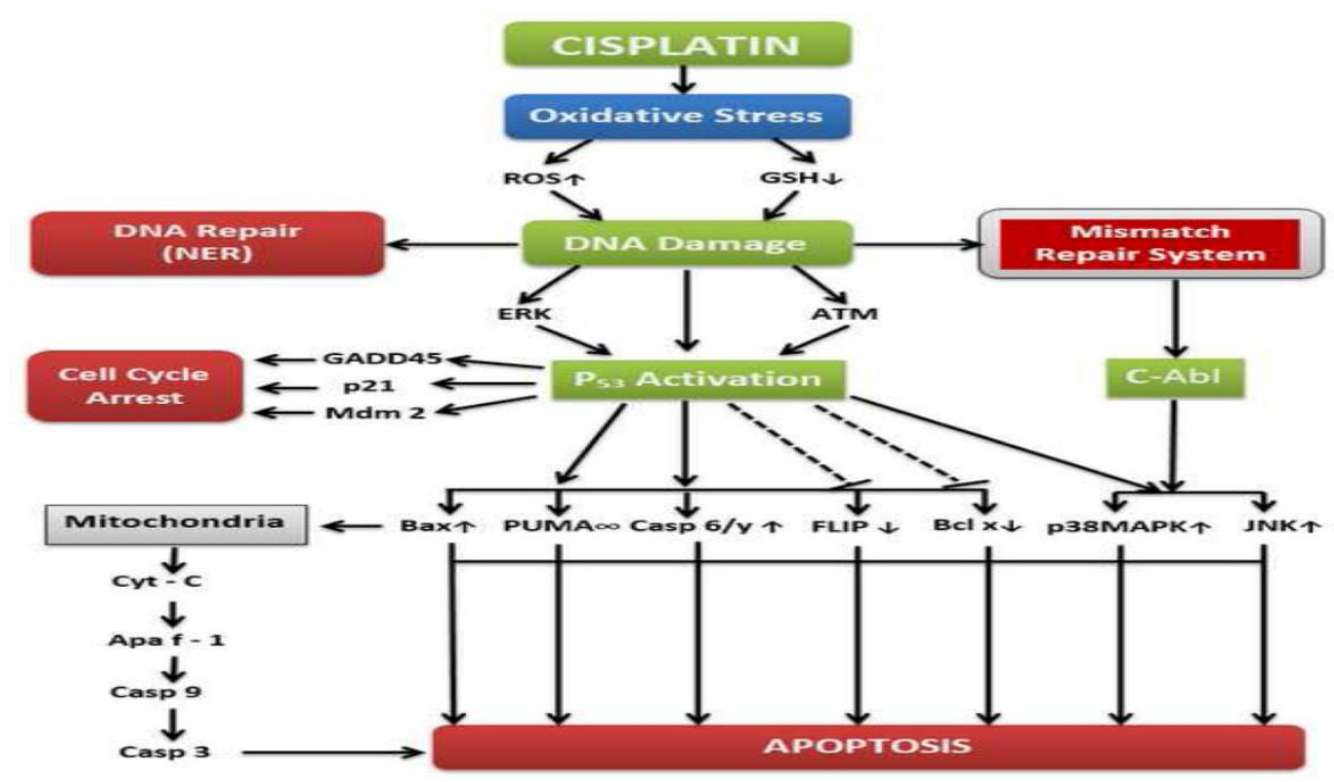

Figure (3): Molecular mechanisms of cisplatin in cancer treatment. ROS: reactive oxygen species, GSH: glutathione, NER: nucleotide excision repair, ERK: extracellular signal-regulated kinase, ATM: ataxia telangiectasia mutated, GADD45: growth arrest and DNA damage 45, Mdm2: mouse double minute 2 homolog, C-Abl: Abelson murine leukemia viral oncogene, Bax: Bcl-2-associated X protein, PUMA: p53 upregulated modulator of apoptosis, Casp: caspase, FLIP: FLICE (FADD-like IL-1 $\beta$-converting enzyme)-inhibitory protein, Bcl xL: B-cell lymphoma extra large, MAPK: mitogen-activated protein kinase, JNK: c-Jun N-terminal kinase, Cyt-C: cytochrome c, Apa f-1: Apoptotic protease activating factor 1 (Dasari et al. 2014).

activity of TQ basiclly depends on three main regulatory pathways: apoptosis, cell cycle, and nuclear factor kappa B (NF- $\mathrm{kB})$. Anti-inflammatory action of TQ is linked to inhibition of proinflammatory cytokine as interleukin 6 (IL-6) and tumor necrosis factor alpha (TNF- $\alpha$ ). TQ excets its free radical scavengering activity by up-regulation of the key anti-oxidant enzymes as glutathione reductase (GR) and glutathione-S-transferase (GST) (Mahmoud et al. 2019). Previous literature outlined the beneficial using of TQ as an adjuvant with the chemotherapeutics and radiation in order to boosts the therapeutic efficacy as well as limiting the undesired toxicity (Mahmoud et al. 2019). Surprisingly, earlier studies reported the inhibitory action of TQ on Notch1, Hes1, and JAG1 and suggested such inhibition is mediated through upregulation of phosphatase and tensin homologue (PTEN) (Ke et al. 2015, Mu et al. 2015). In addition, TQ induces CTNNB1 supression by upregulation of dickkopf Wnt signaling pathway inhibitor 1(DKK1) and cyclin dependent kinase inhibitor 1A (CDNK-1A) (Kensara et al. 2016).

\subsection{Pentoxifylline as a complementary therapy and Notch modulator:}

Pentoxifylline (PTX) is a methylated xanthine derivative drug that was approved for treating peripheral vascular disorders (Namdar et al. 2020). Recent studies revealed the anticancer effect of PTX, which could mediated through apoptosis induction by down-regulation of integrins-adhesion molecules (Niderla-Bielińska et al. 2018) and anti-apoptotic proteins together with up-regulation of death receptors DR4 and DR5. Moreover, PTX impairs DNA-repair mechanis and induces cell cycle arrest at G1-S phase (Talar et al. 2016).

From that point, PTX was used as an adjunct drug to enhances the chemotherapeutic action and ameliorates the undesired side effects. For instance, PTX was used with doxorubicin (Elshazly $\boldsymbol{e t}$ al. 2016) and paclitaxel (Kim et al. 2016) to minimize cardiotoxicity and nephrotoxicity, respictively. Moreover, PTX has been found to supress Notch1 with subsequent Hes1 inhibition (NiderlaBielińska et al. 2018). The anti-inflammatory property could allow PTX to down-regulate JAG1 ligand through inhibition of pro-inflammatory cytokines TNF- $\alpha$ and IL-6 (El-Haggar et al. 2018). 


\section{References:}

Achkar, I. W., Abdulrahman, N., Al-Sulaiti, H., Joseph, J. M., Uddin, S. and Mraiche, F. (2018). Cisplatin based therapy: the role of the mitogen activated protein kinase signaling pathway. J Transl Med 16(1): 96.

Andersen, P., Uosaki, H., Shenje, L. and Kwon, C. (2012). Non-Canonical Notch Signaling: Emerging Role and Mechanism. Trends Cell Biol 22(5): 257265.

Barse, L. and Bocchetta, M. (2015). Non-small-cell lung carcinoma: role of the Notch signaling pathway. Lung Cancer (Auckl) 6: 43-53.

Butti, R., Gunasekaran, V. P., Kumar, T. V. S., Banerjee, P. and Kundu, G. C. (2019). Breast cancer stem cells: Biology and therapeutic implications. Int J Biochem Cell Biol 107: 38-52.

Cao, W.-q., Zhai, X.-q., Ma, J.-w., Fu, X.-q., Zhao, B.-s., Zhang, P. and Fu, X.-y. (2020). Natural borneol sensitizes human glioma cells to cisplatininduced apoptosis by triggering ROS-mediated oxidative damage and regulation of MAPKs and PI3K/AKT pathway. Pharm Biol 58(1): 72-79.

Chatterjee, S. and Sil, P. C. (2019). Targeting the crosstalks of Wnt pathway with Hedgehog and Notch for cancer therapy. Pharmacol Res 142: 251261.

Clara, J. A., Monge, C., Yang, Y. and Takebe, N. (2019). Targeting signalling pathways and the immune microenvironment of cancer stem cells a clinical update. Nat Rev Clin Oncol.

Costa, A. R., Machado, N., Rego, A., Sousa, M. J., Côrte-Real, M. and Chaves, S. R. (2019). Proteasome inhibition prevents cell death induced by the chemotherapeutic agent cisplatin downstream of DNA damage. DNA Repair 73: 2833.

D'Souza, B., Meloty-Kapella, L. and Weinmaster, G. (2010). Canonical and Non-Canonical Notch Ligands. In: R. Kopan, eds. Curr Top Dev Biol, Academic Press. 92: 73-129.

Dasari, S. and Bernard Tchounwou, P. (2014). Cisplatin in cancer therapy: Molecular mechanisms of action. Eur J Pharmacol 740: 364-378.

El-Haggar, S. M., Eissa, M. A., Mostafa, T. M., ElAttar, K. S. and Abdallah, M. S. (2018). The Phosphodiesterase Inhibitor Pentoxifylline as a
Novel Adjunct to Antidepressants in Major Depressive Disorder Patients: A Proof-of-Concept, Randomized, Double-Blind, Placebo-Controlled Trial. Psychother Psychosom 87(6): 331-339.

Elshazly, S. M., Mahmoud, A. A. A. and Barakat, W. (2016). Pentoxifylline abrogates cardiotoxicity induced by the administration of a single high dose or multiple low doses of doxorubicin in rats. Can J Physiol Pharmacol 94(11): 1170-1177.

Ferlay, J., Colombet, M., Soerjomataram, I., Mathers, C., Parkin, D. M., Piñeros, M., Znaor, A. and Bray, F. (2019). Estimating the global cancer incidence and mortality in 2018: GLOBOCAN sources and methods. Int J Cancer 144(8): 19411953.

Ho, D. M., Artavanis-Tsakonas, S. and Louvi, A. (2020). The Notch pathway in CNS homeostasis and neurodegeneration. Wiley Interdiscip Rev Dev Biol 9(1): e358.

IARC. (2018, Last Update Date). The Global Cancer Observatory (GCO). Retrieved Access Date, Access 2018, from https://gco.iarc.fr/today/home.

Ke, X., Zhao, Y., Lu, X., Wang, Z., Liu, Y., Ren, M., Lu, G., Zhang, D., Sun, Z., Xu, Z., Song, J. H., Cheng, Y., Meltzer, S. J. and He, S. (2015). TQ inhibits hepatocellular carcinoma growth in vitro and in vivo via repression of Notch signaling. Oncotarget 6(32): 32610-32621.

Kensara, O. A., El-Shemi, A. G., Mohamed, A. M., Refaat, B., Idris, S. and Ahmad, J. (2016). Thymoquinone subdues tumor growth and potentiates the chemopreventive effect of 5fluorouracil on the early stages of colorectal carcinogenesis in rats. Drug Des Devel Ther 10: 2239-2253.

Kim, H. K., Hwang, S. H., Lee, S. O., Kim, S. H. and Abdi, S. (2016). Pentoxifylline Ameliorates Mechanical Hyperalgesia in a Rat Model of Chemotherapy-Induced Neuropathic Pain. Pain Physician 19(4): E589-600.

Kovall, R. A., Gebelein, B., Sprinzak, D. and Kopan, R. (2017). The Canonical Notch Signaling Pathway: Structural and Biochemical Insights into Shape, Sugar, and Force. Dev Cell 41(3): 228-241.

Mahmoud, Y. K. and Abdelrazek, H. M. A. (2019). Cancer: Thymoquinone antioxidant/pro-oxidant 
effect as potential anticancer remedy. Biomed Pharmacother 115: 108783.

Majdalawieh, A. F. and Fayyad, M. W. (2015). Immunomodulatory and anti-inflammatory action of Nigella sativa and thymoquinone: A comprehensive review. Int Immunopharmacol 28(1): 295-304.

Mu, G.-g., Zhang, L.-1., Li, H.-y., Liao, Y. and Yu, H.-g. (2015). Thymoquinone Pretreatment Overcomes the Insensitivity and Potentiates the Antitumor Effect of Gemcitabine Through Abrogation of Notch1, PI3K/Akt/mTOR Regulated Signaling Pathways in Pancreatic Cancer. Dig Dis Sci 60(4): 1067-1080.

Muggia, F. M., Bonetti, A., Hoeschele, J. D., Rozencweig, M. and Howell, S. B. (2015). Platinum Antitumor Complexes: 50 Years Since Barnett Rosenberg's Discovery. J Clin Oncol 33(35): 42194226.

Namdar, H., Khani, E., Pourrashid, M. H. and Entezari-Maleki, T. (2020). Effects of Adding Pentoxifylline to Captopril on Primary Hypertension: A Pilot Randomized Clinical Trial. $\underline{\mathbf{J}}$ Clin Pharmacol 60(2): 181-187.

Niderla-Bielińska, J., Bartkowiak, K., Ciszek, B., Czajkowski, E., Jankowska-Steifer, E., Krejner, A. and Ratajska, A. (2018). Pentoxifylline inhibits angiogenesis via decreasing Dll4 and Notch1 expression in mouse proepicardial explant cultures. Eur J Pharmacol 827: 80-87.

Qin, X. Y., Wang, Y. N., Liu, H. F., Luo, Z. H., Zhang, P. L., Li-Fang, H. and Liu, M. R. (2020). Anti-cancer activities of metal-based complexes by regulating the VEGF/VEGFR2 signaling pathway and apoptosis-related factors Bcl-2, Bax, and caspase-9 to inhibit angiogenesis and induce apoptosis. Metallomics 12(1): 92-103.

Talar, B., Gajos-Michniewicz, A., Talar, M.,
Chouaib, S. and Czyz, M. (2016). Pentoxifylline Inhibits WNT Signalling in $\beta$-Cateninhigh PatientDerived Melanoma Cell Populations. PLOS ONE 11(6): e0158275.

Tian, W., Shen, J. and Chen, W. (2017). Suppression of midkine gene promotes the antitumoral effect of cisplatin on human gastric cancer cell line AGS in vitro and in vivo via the modulation of Notch signaling pathway. Oncol Rep 38(2): $745-754$.

Xiao, Y.-S., Zeng, D., Liang, Y.-K., Wu, Y., Li, M.-F., Qi, Y.-Z., Wei, X.-L., Huang, W.-H., Chen, M. and Zhang, G.-J. (2019). Major vault protein is a direct target of Notch1 signaling and contributes to chemoresistance in triple-negative breast cancer cells. Cancer Lett 440-441: 156-167.

Yin, P., Song, G. and Jiang, Z. (2018). Cisplatin suppresses proliferation, migration and invasion of nasopharyngeal carcinoma cells in vitro by repressing the $\mathrm{Wnt} / \beta$-catenin/Endothelin-1 axis via activating $\mathrm{B}$ cell translocation gene 1. Cancer Chemother Pharmacol 81(5): 863-872.

Yuan, X., Wu, H., Xu, H., Xiong, H., Chu, Q., Yu, S., Wu, G. S. and Wu, K. (2015). Notch signaling: An emerging therapeutic target for cancer treatment. Cancer Lett 369(1): 20-27.

Zidan, A.-A. A., El-Ashmawy, N. E., Khedr, E. G., Ebeid, E.-Z. M., Salem, M. L. and Mosalam, E. M. (2018). Loading of doxorubicin and thymoquinone with F2 gel nanofibers improves the antitumor activity and ameliorates doxorubicin-associated nephrotoxicity. Life Sci 207: 461-470.

Zlobin, A., Bloodworth, J. C., Baker, A. T. and Osipo, C. (2019). Notch Signaling Pathway in Carcinogenesis. In: S. Badve and G. L. Kumar, eds. Predictive Biomarkers in Oncology: Applications in Precision Medicine. Cham, Springer International Publishing: 223-230. 